\title{
An Application of Neutrosophic Bipolar Vague On Multi-Criteria Decision Making Problems
}

\author{
${ }^{1}$ Princy R, ${ }^{2}$ Mohana K \\ ${ }^{1}$ Research Scholar, ${ }^{2}$ Assistant Professor \\ Nirmala College For Women, Coimbatore, India. \\ ${ }^{1}$ princy.pjs@gmail.com, ${ }^{2}$ riyaraju1116@gmail.com
}

\begin{abstract}
In this paper, we studied the concept of neutrosophic bipolar vague set and some of its operations. Also, we propose score, certainty and accuracy functions to compare the neutrosophic bipolar vague sets. Then, we develop the neutrosophic bipolar vague weighted average operator $\left(A_{\omega}\right)$ and neutrosophic bipolar vague weighted geometric operator $\left(G_{\omega}\right)$ to aggregate the neutrosophic bipolar vague information. Furthermore, based on the $\left(A_{\omega}\right)$ and $\left(G_{\omega}\right)$ operators and the score, certainty and accuracy functions, we develop a neutrosophic bipolar vague multiple criteria decision-making approach, in which the evaluation values of alternatives on the attributes take the form of neutrosophic bipolar vague numbers to select the most desirable one(s). Finally, numerical examples of the method were given to demonstrate the application and effectiveness of the developed method.
\end{abstract}

Keywords- Neutrosophic set, neutrosophic bipolar vague set, average operator, geometric operator, score, certainty and accuracy functions, multi-criteria decision making.

\section{INTRODUCTION:}

To handle with imprecision and uncertainty, concept of fuzzy sets and intuitionistic fuzzy sets originally introduced by Zadeh [30] and Atanassov [1], respectively. Then, Smarandache [21] proposed concept of neutrosophic set which is generalization of fuzzy set theory and intuitionistic fuzzy sets. These sets models have been studied by many authors; on application $[4,6,8,12-14,18,19]$, theory [22-24,25-29,31,32], and so on. Bosc and Pivert [2] said that "Bipolarity refers to the propensity of the human mind to reason and make decisions on the basis of positive and negative affects. Positive information states what is possible, satisfactory, permitted, desired, or considered as being acceptable. On the other hand, negative statements express what is impossible, rejected, or forbidden. Negative preferences correspond to constraints, since they specify which values or objects have to be rejected (i.e., those that do not satisfy the constraints), while positive preferences correspond to wishes, as they specify which objects

are more desirable than others (i.e., satisfy user wishes) without rejecting those that do not meet the wishes." Therefore, Lee [10,11] introduced the concept of bipolar fuzzy sets which is an generalization of the fuzzy sets. Neutrosophic vague set is a combination of neutrosophic set and vague set which was defined by Shawkat Alkhazaleh[20].
In this paper, we introduced the concept of neutrosophic bipolar vague sets which is an extension of the fuzzy sets, bipolar fuzzy sets, intuitionistic fuzzy sets, neutrosophic sets and vague sets. Also, we give some operations and operators on the neutrosophic bipolar vague. The operations and operators generalizes the operations and operators of fuzzy sets, bipolar fuzzy sets, intuitionistic fuzzy sets, neutrosophic sets and vague sets which has been previously proposed. Therefore, in section 2, we studied the concept of neutrosophic bipolar vague set and its some of its operations. In section 3, we introduced the concept of score, certainity and accuracy funtions of the neutrosophic bipolar vague sets for comparision. In the same section, we also develop the neutrosophic bipolar vague weighted average operator $\left(A_{\omega}\right)$ and neutrosophic bipolar vague weighted geometric operator $\left(G_{\omega}\right)$ operator to aggregate the neutrosophic bipolar vague information. In section 4 , based on the $\left(A_{\omega}\right)$ and $\left(G_{\omega}\right)$ operators and the score, certainty and accuracy functions, we develop a neutrosophic bipolar vague multiple criteria decision-making approach, in which the evaluation values of alternatives on the attributes take the form of neutrosophic bipolar vague numbers to select the most desirable one(s) and give numerical examples to demonstrate the application and effectiveness of the developed method.

\section{PRELIMINARIES}

Definition 2. 1[10,11]: Let $X$ be the universe. Then a bipolar valued fuzzy sets, $\mathrm{A}$ on $\mathrm{X}$ is defined by 


\section{Available online at www.ijrat.org}

positive membership function $\mu_{A}^{+}: \mathrm{X} \rightarrow[0,1]$ and a negative membership function $\mu_{A}^{-}: \mathrm{X} \rightarrow[-1,0]$. For sake of simplicity, we shall use the symbol $A=\{<x$, $\left.\mu_{A}^{+}(\mathrm{x}), \mu_{A}^{-}(\mathrm{x})>: \mathrm{x} \in \mathrm{X}\right\}$.

Definition 2. 2[10,11]: Let A and B be two bipolar valued fuzzy sets then their union, intersection and complement are defined as follows:

(i) $\mu_{A \cup B}^{+}(x)=\max \left\{\mu_{A}^{+}(\mathrm{x}), \mu_{B}^{+}(\mathrm{x})\right\}$.

(ii) $\mu_{A \cup B}^{-}(x)=\min \left\{\mu_{A}^{-}(\mathrm{x}), \mu_{B}^{-}(\mathrm{x})\right\}$.

(iii) $\mu_{A \cap B}^{+}(x)=\min \left\{\mu_{A}^{+}(\mathrm{x}), \mu_{B}^{+}(\mathrm{x})\right\}$.

(iv) $\mu_{A \cap B}^{-}(x)=\max \left\{\mu_{A}^{-}(\mathrm{x}), \mu_{B}^{-}(\mathrm{x})\right\}$.

(v) $\mu_{\bar{A}}^{+}(x)=1-\mu_{A}^{+}(\mathrm{x})$ and $\mu_{\bar{A}}^{-}(x)=-1-\mu_{A}^{+}(\mathrm{x})$ for all $\mathrm{x} \in X$.

Definition 2.3(5): A vague set $A$ in the universe of discourse $U$ is a pair $\left(t_{A}, f_{A}\right)$ where $t_{A}: U \rightarrow[0,1], f_{A}$ $: U \rightarrow[0,1]$ are the mapping such that $t_{A}+f_{A} \leq 1$ for all $\mathrm{u} \in U$.The function $\mathrm{t}_{\mathrm{A}}$ and $\mathrm{f}_{\mathrm{A}}$ are called true membership function and false membership function respectively. The interval $\left[\mathrm{t}_{\mathrm{A}}, 1-\mathrm{f}_{\mathrm{A}}\right]$ is called the vague value of $\mathrm{u}$ in $\mathrm{A}$, and denoted by $v_{A}(u)$, i.e $v_{A}(u)=\left[t_{A}, 1-f_{A}\right]$.

Definition 2.4[5]: Let $A$ be a non-empty set and the vague set $A$ and $B$ in the form $A=\left\{\left\langle x, t_{A}, 1-f_{A}\right.\right.$ $>: \mathrm{x} \in X\}, \mathrm{B}=\left\{\left\langle\mathrm{x}, \mathrm{t}_{\mathrm{B}}, 1-\mathrm{f}_{\mathrm{B}}>: \mathrm{x} \in X\right\}\right.$.

Then,

(i) $A \subseteq B$ if and only if $t_{A}(x) \leq t_{A}(x)$ and $1-f_{B}(x) \leq 1-$ $f_{B}(x)$.

(ii) $\mathrm{A} \cup \quad \mathrm{B}=\left\{<\max \left(\mathrm{t}_{\mathrm{A}}(\mathrm{x}), \mathrm{t}_{\mathrm{B}}(\mathrm{x})\right), \quad \max \left(1-\mathrm{f}_{\mathrm{A}}(\mathrm{x}), 1-\right.\right.$ $\left.\left.f_{B}(x)\right)>/ x \in X\right\}$.

(iii) $\mathrm{A} \cap \mathrm{B}=\left\{<\min \left(\mathrm{t}_{\mathrm{A}}(\mathrm{x}), \mathrm{t}_{\mathrm{B}}(\mathrm{x})\right), \quad \min \left(1-\mathrm{f}_{\mathrm{A}}(\mathrm{x}), 1-\right.\right.$ $\left.\left.\mathrm{f}_{\mathrm{B}}(\mathrm{x})\right)>/ \mathrm{x} \in \mathrm{X}\right\}$.

(iv) $\bar{A}=\left\{\left\langle\mathrm{x}, \mathrm{f}_{\mathrm{A}}(\mathrm{x}), 1-\mathrm{t}_{\mathrm{A}}(\mathrm{x})>\mathrm{x} \in \mathrm{X}\right\}\right.$.

Definition 2.5[21]: Let $X$ be a universe of discourse. Then a neutrosophic set is defined as:

$A=\left\{\left\langle\mathrm{x}, \mathrm{T}_{\mathrm{A}}(\mathrm{x}), \mathrm{I}_{\mathrm{A}}(\mathrm{x}), \mathrm{F}_{\mathrm{A}}(\mathrm{x})\right\rangle: \mathrm{x} \in \mathrm{X}\right\}$, which is characterized by a truth-membership function $\mathrm{T}_{\mathrm{A}}: \mathrm{X}$ $\rightarrow] 0-, 1+[$, an indeterminacy membership function $\left.\mathrm{I}_{\mathrm{A}}: \mathrm{X} \rightarrow\right] 0^{-}, 1+[$ and a falsity-membership function $\left.\mathrm{F}_{\mathrm{A}}: \mathrm{X} \rightarrow\right] 0^{-}, 1+[$. There is not restriction on the sum of $\mathrm{T}_{\mathrm{A}}(\mathrm{x}), \mathrm{I}_{\mathrm{A}}(\mathrm{x})$ and $\mathrm{F}_{\mathrm{A}}(\mathrm{x})$, so $0^{-} \leq \sup _{\mathrm{A}}(\mathrm{x}) \leq$ $\operatorname{supI}_{\mathrm{A}}(\mathrm{x}) \leq \operatorname{supF}_{\mathrm{A}}(\mathrm{x}) \leq 3+$.

Definition 2.6[17]: A neutrosophic bipolar vague set $\mathrm{A}$ in $\mathrm{X}$ is defined as an object of the form,

$$
\begin{aligned}
& \mathrm{A}=\{< \\
& \mathrm{x}, \\
& {\left[T^{-}(x), T^{+}(x)\right]^{+},\left[I^{-}(x), I^{+}(x)\right]^{+},\left[F^{-}(x), F^{+}(x)\right]^{+},} \\
& {\left[T^{-}(x), T^{+}(x)\right]^{-},\left[I^{-}(x), I^{+}(x)\right]^{-},\left[F^{-}(x), F^{+}(x)\right]^{-}} \\
& >: \mathrm{x} \in \mathrm{X}\}, \text { where } \\
& T^{+}=1-F^{-}, F^{+}=1-T^{-}, \text {and } \\
& T^{+}, I^{+}, F^{+}: \mathrm{X} \rightarrow[2,0] \text { and } T^{-}, I^{-}, F^{-}: \mathrm{X} \rightarrow[-2,0] .
\end{aligned}
$$

The positive membership degree $\left[T^{-}(x), T^{+}(x)\right]^{+},\left[I^{-}(x), I^{+}(x)\right]^{+},\left[F^{-}(x), F^{+}(x)\right]^{+}$ denotes the truth membership, indeterminate membership and false membership of an element $\mathrm{x} \in \mathrm{X}$ corresponding to a neutrosophic bipolar vague set $A$ and the negative membership degree $\left[T^{-}(x), T^{+}(x)\right]^{-},\left[I^{-}(x), I^{+}(x)\right]^{-},\left[F^{-}(x), F^{+}(x)\right]^{-}$ denotes the truth membership, indeterminate membership and false membership of an element $\mathrm{x} \in \mathrm{X}$ to some implicit counter-property corresponding to a neutrosophic bipolar vague set A.

Definition 2.7[17]: Let $A=\{<\quad x$, $\left[T_{A}^{-}, T_{A}^{+}\right]^{+},\left[I_{A}^{-}, I_{A}^{+}\right]^{+},\left[F_{A}^{-}, F_{A}^{+}\right]^{+},\left[T_{A}^{-}, T_{A}^{+}\right]^{-},\left[I_{A}^{-}, I_{A}^{+}\right]^{-}$, $\left.\left[F_{A}^{-}, F_{A}^{+}\right]^{->}\right\}$and

$\mathrm{B}=\{<$ $\mathrm{x}$, $\left[T_{B}^{-}, T_{B}^{+}\right]^{+},\left[I_{B}^{-}, I_{B}^{+}\right]^{+},\left[F_{B}^{-}, F_{B}^{+}\right]^{+},\left[T_{B}^{-}, T_{B}^{+}\right]^{-},\left[I_{B}^{-}, I_{B}^{+}\right]^{-}$, $\left.\left[F_{B}^{-}, F_{B}^{+}\right]^{-}>\right\}$be two neutrosophic bipolar vague sets. Then $\mathrm{A} \subseteq \mathrm{B}$ if and only if

$\left[T_{A}^{-} \leq T_{B}^{-}\right]^{+},\left[T_{A}^{+} \leq T_{B}^{+}\right]^{+},\left[I_{A}^{-} \geq I_{B}^{-}\right]^{+},\left[I_{A}^{+} \geq I_{B}^{+}\right]^{+}$, $\left[F_{A}^{-} \geq F_{B}^{-}\right]^{+},\left[F_{A}^{+} \geq F_{B}^{+}\right]^{+}$, and

$\left[T_{A}^{-} \geq T_{B}^{-}\right]^{-},\left[T_{A}^{+} \geq T_{B}^{+}\right]^{-}, \quad\left[I_{A}^{-} \leq I_{B}^{-}\right]^{-},\left[I_{A}^{+} \leq\right.$ $\left.I_{B}^{+}\right]^{-},\left[F_{A}^{-} \leq F_{B}^{-}\right]^{-},\left[F_{A}^{+} \leq F_{B}^{+}\right]^{-}$for all $\mathrm{x} \in \mathrm{X}$.

Definition 2.8[17]: Let $\mathrm{A}=\{<\quad \mathrm{X}$, $\left[T_{A}^{-}, T_{A}^{+}\right]^{+},\left[I_{A}^{-}, I_{A}^{+}\right]^{+},\left[F_{A}^{-}, F_{A}^{+}\right]^{+},\left[T_{A}^{-}, T_{A}^{+}\right]^{-},\left[I_{A}^{-}, I_{A}^{+}\right]^{-},\left[F_{A}^{-}, F_{A}^{+}\right]^{-}$ $>\} \quad$ and $\mathrm{B}=\{<\quad \mathrm{x}$, $\left[T_{B}^{-}, T_{B}^{+}\right]^{+},\left[I_{B}^{-}, I_{B}^{+}\right]^{+},\left[F_{B}^{-}, F_{B}^{+}\right]^{+},\left[T_{B}^{-}, T_{B}^{+}\right]^{-}$,

$\left.\left[I_{B}^{-}, I_{B}^{+}\right]^{-},\left[F_{B}^{-}, F_{B}^{+}\right]^{-}>\right\}$be two neutrosophic bipolar vague sets. Then $\mathrm{A}=\mathrm{B}$ if and only if $\left[T_{A}^{-}=T_{B}^{-}\right]^{+}$, $\left[T_{A}^{+}=T_{B}^{+}\right]^{+}, \quad\left[I_{A}^{-}=I_{B}^{-}\right]^{+}, \quad\left[I_{A}^{+}=I_{B}^{+}\right]^{+}, \quad\left[F_{A}^{-}=\right.$ $\left.F_{B}^{-}\right]^{+},\left[F_{A}^{+}=F_{B}^{+}\right]^{+}$, and $\left[T_{A}^{-}=T_{B}^{-}\right]^{-},\left[T_{A}^{+}=T_{B}^{+}\right]^{-}$, $\left[I_{A}^{-}=I_{B}^{-}\right]^{-},\left[I_{A}^{+}=I_{B}^{+}\right]^{-},\left[F_{A}^{-}=F_{B}^{-}\right]^{-}, \quad\left[F_{A}^{+}=F_{B}^{+}\right]^{-}$ for all $\mathrm{x} \in \mathrm{X}$.

Definition 2.9[17]: Let $\mathrm{A}=\{<\quad \mathrm{X}$, $\left[T_{A}^{-}, T_{A}^{+}\right]^{+},\left[I_{A}^{-}, I_{A}^{+}\right]^{+},\left[F_{A}^{-}, F_{A}^{+}\right]^{+},\left[T_{A}^{-}, T_{A}^{+}\right]^{-}$,

$\left.\left[I_{A}^{-}, I_{A}^{+}\right]^{-},\left[F_{A}^{-}, F_{A}^{+}\right]^{-}>\right\} \quad$ and $\quad \mathrm{B}=\{<\quad \mathrm{X}$, $\left[T_{B}^{-}, T_{B}^{+}\right]^{+},\left[I_{B}^{-}, I_{B}^{+}\right]^{+},\left[F_{B}^{-}, F_{B}^{+}\right]^{+},\left[T_{B}^{-}, T_{B}^{+}\right]^{-}$,

$\left.\left[I_{B}^{-}, I_{B}^{+}\right]^{-},\left[F_{B}^{-}, F_{B}^{+}\right]^{-}>\right\}$be two neutrosophic bipolar vague sets. Then their union is defined as: 


\section{Available online at www.ijrat.org}

$\mathrm{A} \cup \mathrm{B}=\left\{\max \left[T_{A}^{-}, T_{B}^{-}\right]^{+}, \max \left[T_{A}^{+}, T_{B}^{+}\right]^{+}\right.$,

$\min \left[I_{A}^{-}, I_{B}^{-}\right]^{+}, \min \left[I_{A}^{+}, I_{B}^{+}\right]^{+}$,

$\min \left[F_{A}^{-}, F_{B}^{-}\right]^{+}, \min \left[F_{A}^{+}, F_{B}^{+}\right]^{+}$,

$\min \left[T_{A}^{-}, T_{B}^{-}\right]^{-}, \min \left[T_{A}^{+}, T_{B}^{+}\right]^{-}$,

$\max \left[I_{A}^{-}, I_{B}^{-}\right]^{-}, \max \left[I_{A}^{+}, I_{B}^{+}\right]^{-}$,

$\left.\max \left[F_{A}^{-}, F_{B}^{-}\right]^{-}, \quad \max \left[F_{A}^{+}, F_{B}^{+}\right]^{-}\right\}$for all

$\mathrm{x} \in \mathrm{X}$.

Definition 2.10[17]: Let $A=\{<\quad X$, $\left[T_{A}^{-}, T_{A}^{+}\right]^{+},\left[I_{A}^{-}, I_{A}^{+}\right]^{+},\left[F_{A}^{-}, F_{A}^{+}\right]^{+},\left[T_{A}^{-}, T_{A}^{+}\right]^{-},\left[I_{A}^{-}, I_{A}^{+}\right]^{-},\left[F_{A}^{-}, F_{A}^{+}\right]^{-}$ $>\}$ and

$\mathrm{B}=\{<\mathrm{x}$,

$\left[T_{B}^{-}, T_{B}^{+}\right]^{+},\left[I_{B}^{-}, I_{B}^{+}\right]^{+},\left[F_{B}^{-}, F_{B}^{+}\right]^{+},\left[T_{B}^{-}, T_{B}^{+}\right]^{-},\left[I_{B}^{-}, I_{B}^{+}\right]^{-},\left[F_{B}^{-}, F_{B}^{+}\right]^{-}$ $>$ \} be two neutrosophic bipolar vague sets. Then their intersection is defined as:

$\mathrm{A} \cap \mathrm{B}=\left\{\min \left[T_{A}^{-}, T_{B}^{-}\right]^{+}, \min \left[T_{A}^{+}, T_{B}^{+}\right]^{+}\right.$,

$\max \left[I_{A}^{-}, I_{B}^{-}\right]^{+}, \max \left[I_{A}^{+}, I_{B}^{+}\right]^{+}$,

$\max \left[F_{A}^{-}, F_{B}^{-}\right]^{+}, \max \left[F_{A}^{+}, F_{B}^{+}\right]^{+}$,

$\max \left[T_{A}^{-}, T_{B}^{-}\right]^{-}, \max \left[T_{A}^{+}, T_{B}^{+}\right]^{-}$,

$\min \left[I_{A}^{-}, I_{B}^{-}\right]^{-}, \min \left[I_{A}^{+}, I_{B}^{+}\right]^{-}$,

$\left.\min \left[F_{A}^{-}, F_{B}^{-}\right]^{-}, \min \left[F_{A}^{+}, F_{B}^{+}\right]^{-}\right\}$for all $\mathrm{x} \in \mathrm{X}$.

Definition 2.11[17]: Let $A=\{<\quad x$, $\left[T_{A}^{-}, T_{A}^{+}\right]^{+},\left[I_{A}^{-}, I_{A}^{+}\right]^{+},\left[F_{A}^{-}, F_{A}^{+}\right]^{+},\left[T_{A}^{-}, T_{A}^{+}\right]^{-},\left[I_{A}^{-}, I_{A}^{+}\right]^{-},\left[F_{A}^{-}, F_{A}^{+}\right]^{-}$ $>: x \in X\}$ be a neutrosophic bipolar vague set in $\mathrm{X}$. Then the complement of $\mathrm{A}$ is denoted by $\bar{A}$ and is defined by, $\bar{A}=\left\{<\left[F_{A}^{-}, F_{A}^{+}\right]^{+},\left[1-I_{A}^{-}, 1-\right.\right.$ $\left.I_{A}^{+}\right]^{+},\left[T_{A}^{-}, T_{A}^{+}\right]^{+},\left[F_{A}^{-}, F_{A}^{+}\right]^{-},\left[1-I_{A}^{-}, 1-\right.$

$\left.\left.I_{A}^{+}\right]^{-},\left[T_{A}^{-}, T_{A}^{+}\right]^{-}>\right\}$for all $\mathrm{x} \in \mathrm{X}$.

\section{NEUTROSOPHIC BIPOLAR VAGUE NUMBER}

Definition

3.1:

Let

$\widetilde{a_{1}}=<\left[T_{1}{ }^{-}, T_{1}{ }^{+}\right]^{+},\left[{I_{1}}^{-}, I_{1}{ }^{+}\right]^{+},\left[F_{1}{ }^{-},{F_{1}}^{+}\right]^{+}$,

$\left[{T_{1}}^{-}, T_{1}{ }^{+}\right]^{-},\left[{I_{1}}^{-}, I_{1}{ }^{+}\right]^{-},\left[{F_{1}}^{-},{F_{1}}^{+}\right]^{-}>$and $\widetilde{a_{2}}=<$ $\left[\mathrm{T}_{2}{ }^{-}, \mathrm{T}_{2}{ }^{+}\right]^{+},\left[\mathrm{I}_{2}{ }^{-}, \mathrm{I}_{2}{ }^{+}\right]^{+},\left[\mathrm{F}_{2}{ }^{-},{F_{2}}^{+}\right]^{+}$,

$\left[\mathrm{T}_{2}{ }^{-}, \mathrm{T}_{2}{ }^{+}\right]^{-},\left[\mathrm{I}_{2}{ }^{-}, \mathrm{I}_{2}{ }^{+}\right]^{-},\left[\mathrm{F}_{2}{ }^{-},{F_{2}}^{+}\right]^{-}>$be two neutrosophic bipolar vague numbers. Then the operations for NBVNs are defined as below:

$$
\text { i. } \begin{aligned}
& \lambda \widetilde{a_{1}}=< \\
& 1- \\
&\left(1-\left[T_{1}{ }^{-}, T_{1}{ }^{+}\right]^{+}\right)^{\lambda},\left(\left[I_{1}{ }^{-}, I_{1}{ }^{+}\right]^{+}\right)^{\lambda}, \\
&\left(\left[{F_{1}}^{-},{F_{1}}^{+}\right]^{+}\right)^{\lambda},-\left(-\left[{T_{1}}^{-},{T_{1}}^{+}\right]^{-}\right)^{\lambda}, \\
& \\
&-\left(-\left[I_{1}{ }^{-}, I_{1}{ }^{+}\right]^{-}\right)^{\lambda},
\end{aligned}
$$

$$
-\left(1-\left(1-\left(-\left[{F_{1}}^{-},{F_{1}}^{+}\right]^{-}\right)\right)^{\lambda}\right)>
$$

ii.

$$
\begin{aligned}
& {\widetilde{a_{1}}}^{\lambda}=<\left(\left[T_{1}{ }^{-}, T_{1}{ }^{+}\right]^{+}\right)^{\lambda}, 1- \\
& \left(1-\left[I_{1}{ }^{-}, I_{1}{ }^{+}\right]^{+}\right)^{\lambda}, 1-(1- \\
& \left.\left[F_{1}{ }^{-}, F_{1}{ }^{+}\right]^{+}\right)^{\lambda},-(1-(1- \\
& \left.\left.\left(-\left[{T_{1}}^{-}, T_{1}{ }^{+}\right]^{-}\right)\right)^{\lambda}\right),-\left(-\left[I_{1}{ }^{-}, I_{1}{ }^{+}\right]^{-}\right)^{\lambda}, \\
& \left.-\left(-F^{-}, F^{+}\right]^{-}\right)^{\lambda}>
\end{aligned}
$$

iii. $\quad \widetilde{a_{1}}+\widetilde{a_{2}}=<\left[T_{1}{ }^{-}, T_{1}^{+}\right]^{+}+$

$$
\left[T_{2}{ }^{-}, T_{2}{ }^{+}\right]^{+}-
$$$$
\left[T_{1}{ }^{-}, T_{1}{ }^{+}\right]^{+} \cdot\left[T_{2}{ }^{-}, T_{2}{ }^{+}\right]^{+} \text {, }
$$$$
\left[I_{1}{ }^{-}, I_{1}{ }^{+}\right]^{+} \cdot\left[I_{2}{ }^{-}, I_{2}{ }^{+}\right]^{+} \text {, }
$$$$
\left[F_{1}{ }^{-}, F_{1}{ }^{+}\right]^{+} \cdot\left[F_{2}{ }^{-}, F_{2}{ }^{+}\right]^{+} \text {, }
$$$$
-\left(\left[{T_{1}}^{-}, T_{1}^{+}\right]^{-} \cdot\left[T_{2}{ }^{-}, T_{2}{ }^{+}\right]^{-}\right),
$$

$-\left(-\left[I_{1}{ }^{-}, I_{1}{ }^{+}\right]^{-}-\left[I_{2}{ }^{-}, I_{2}{ }^{+}\right]^{-}-\right.$

$\left.\left[I_{1}{ }^{-}, I_{1}{ }^{+}\right]^{-} \cdot\left[I_{2}{ }^{-}, I_{2}{ }^{+}\right]^{-}\right),-\left(-\left[F_{1}{ }^{-}, F_{1}{ }^{+}\right]^{-}-\right.$

$\left.\left[F_{2}{ }^{-},{F_{2}}^{+}\right]^{-}-\left[F_{1}{ }^{-},{F_{1}}^{+}\right]^{-} \cdot\left[{F_{2}}^{-},{F_{2}}^{+}\right]^{-}\right)>$

iv.

$$
\begin{aligned}
& \widetilde{a_{1}} \cdot \widetilde{a_{2}}=< \\
& {\left[T_{1}{ }^{-}, T_{1}{ }^{+}\right]^{+} \cdot\left[T_{2}{ }^{-}, T_{2}{ }^{+}\right]^{+},\left[I_{1}{ }^{-}, I_{1}{ }^{+}\right]^{+}+} \\
& {\left[I_{2}{ }^{-}, I_{2}{ }^{+}\right]^{+}-} \\
& {\left[I_{1}{ }^{-}, I_{1}{ }^{+}\right]^{+} \cdot\left[I_{2}{ }^{-}, I_{2}{ }^{+}\right]^{+},\left[F_{1}{ }^{-}, F_{1}{ }^{+}\right]^{+}+} \\
& {\left[F_{2}{ }^{-}, F_{2}{ }^{+}\right]^{+}-} \\
& {\left[F_{1}{ }^{-}, F_{1}{ }^{+}\right]^{+} \cdot\left[F_{2}{ }^{-}, F_{2}{ }^{+}\right]^{+},-\left(-\left[T_{1}{ }^{-}, T_{1}{ }^{+}\right]^{-}-\right.} \\
& {\left[T_{2}{ }^{-}, T_{2}{ }^{+}\right]^{-}-} \\
& \left.\left[T_{1}{ }^{-}, T_{1}{ }^{+}\right]^{-} \cdot\left[T_{2}{ }^{-}, T_{2}{ }^{+}\right]^{-}\right),
\end{aligned}
$$

$$
-\left(\left[{I_{1}}^{-}, I_{1}{ }^{+}\right]^{-} \cdot\left[I_{2}{ }^{-}, I_{2}{ }^{+}\right]^{-}\right),-\left(\left[F_{1}{ }^{-},{F_{1}}^{+}\right]^{-} \cdot\left[{F_{2}}^{-},{F_{2}}^{+}\right]^{-}\right)
$$$$
>
$$

Where $\lambda>0$.

Definition 3.2: Let $\widetilde{a_{1}}=$ $<\left[T_{1}{ }^{-}, T_{1}{ }^{+}\right]^{+},\left[I_{1}{ }^{-}, I_{1}{ }^{+}\right]^{+},\left[{F_{1}}^{-}, F_{1}{ }^{+}\right]^{+}$,

$\left[{T_{1}}^{-}, T_{1}{ }^{+}\right]^{-},\left[I_{1}{ }^{-}, I_{1}{ }^{+}\right]^{-},\left[{F_{1}}^{-},{F_{1}}^{+}\right]^{-}>$be a neutrosophic bipolar vague number. Then, the score function $s\left(\widetilde{a_{1}}\right)$, accuracy function $a\left(\widetilde{a_{1}}\right)$, and certainty function $c\left(\widetilde{a_{1}}\right)$ of an NBVN are defined as follows:

i. $\quad \tilde{s}\left(\widetilde{a_{1}}\right)=$

ii. $\quad \tilde{a}\left(\widetilde{a_{1}}\right)=\left[T_{1}{ }^{-}, T_{1}{ }^{+}\right]^{+}-\left[F_{1}{ }^{-}, F_{1}{ }^{+}\right]^{+}+$

$\left\{\begin{array}{c}{\left[T_{1}{ }^{-}, T_{1}{ }^{+}\right]^{+}+\left(1-\left[I_{1}{ }^{-}, I_{1}{ }^{+}\right]^{+}\right)+} \\ \left(1-\left[{F_{1}}^{-}, F_{1}{ }^{+}\right]^{+}\right)+\left(1+\left[T_{1}{ }^{-}, T_{1}{ }^{+}\right]^{-}\right)- \\ {\left[I_{1}{ }^{-}, I_{1}{ }^{+}\right]^{-}-\left[{F_{1}}^{-},{F_{1}}^{+}\right]^{-}}\end{array}\right\} / 6$

$\left[T_{1}^{-}, T_{1}^{+}\right]^{-}-\left[F_{1}{ }^{-}, F_{1}^{+}\right]$

iii. $\quad \tilde{c}\left(\widetilde{\left.a_{1}\right)}=\left[T_{1}{ }^{-}, T_{1}^{+}\right]^{+}-\left[F_{1}{ }^{-}, F_{1}{ }^{+}\right]^{-}\right.$

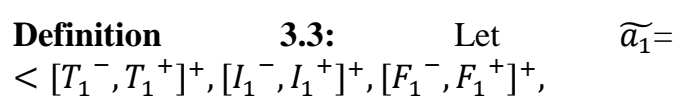




\section{Available online at www.ijrat.org}

$\left[{T_{1}}^{-},{T_{1}}^{+}\right]^{-},\left[{I_{1}}^{-}, I_{1}^{+}\right]^{-},\left[{F_{1}}^{-},{F_{1}}^{+}\right]^{-}>$and $\widetilde{a_{2}}=<\left[T_{2}{ }^{-}, T_{2}{ }^{+}\right]^{+},\left[I_{2}{ }^{-}, I_{2}{ }^{+}\right]^{+},\left[F_{2}{ }^{-}, F_{2}{ }^{+}\right]^{+}$,

$\left[\mathrm{T}_{2}{ }^{-}, \mathrm{T}_{2}{ }^{+}\right]^{-},\left[\mathrm{I}_{2}{ }^{-}, \mathrm{I}_{2}{ }^{+}\right]^{-},\left[\mathrm{F}_{2}{ }^{-}, \mathrm{F}_{2}{ }^{+}\right]^{-}>$be two neutrosophic bipolar vague number. The comparison method can be defined as follows:

i. If $\tilde{s}\left(\widetilde{a_{1}}\right)>\tilde{s}\left(\widetilde{a_{2}}\right)$, then $\widetilde{a_{1}}$ is greater than $\widetilde{a_{2}}$, that is, $\widetilde{a_{1}}$ is superior to $\widetilde{a_{2}}$, denoted by $\widetilde{a_{1}}>\widetilde{a_{2}}$.

ii. If $\tilde{s}\left(\widetilde{a_{1}}\right)=\tilde{s}\left(\widetilde{a_{2}}\right)$ and $\tilde{a}\left(\widetilde{a_{1}}\right)>\tilde{a}\left(\widetilde{a_{2}}\right)$ then $\widetilde{a_{1}}$ is greater than $\widetilde{a_{2}}$, that is, $\widetilde{a_{1}}$ is superior to $\widetilde{a_{2}}$, denoted by $\widetilde{a_{1}}>\widetilde{a_{2}}$.

iii. If $\tilde{s}\left(\widetilde{a_{1}}\right)=\tilde{s}\left(\widetilde{a_{2}}\right), \tilde{a}\left(\widetilde{a_{1}}\right)=\tilde{a}\left(\widetilde{a_{2}}\right)$ and $\tilde{c}\left(\widetilde{a_{1}}\right)>\tilde{c}\left(\widetilde{a_{2}}\right)$, then $\widetilde{a_{1}}$ is greater than $\widetilde{a_{2}}$, that is, $\widetilde{a_{1}}$ is superior to $\widetilde{a_{2}}$, denoted by $\widetilde{a_{1}}>\widetilde{a_{2}}$.

iv. If $\tilde{s}\left(\widetilde{a_{1}}\right)=\tilde{s}\left(\widetilde{a_{2}}\right), \tilde{a}\left(\widetilde{a_{1}}\right)=\tilde{a}\left(\widetilde{a_{2}}\right)$ and $\tilde{c}\left(\widetilde{a_{1}}\right)=\tilde{c}\left(\widetilde{a_{2}}\right)$, then $\widetilde{a_{1}}$ is equal to $\widetilde{a_{2}}$, that is, $\widetilde{a_{1}}$ is indifferent to $\widetilde{a_{2}}$, denoted by $\widetilde{a_{1}}=\widetilde{a_{2}}$.

Based on the study given in [15,21 ] we define some weighted aggregation operators related to neutrosophic bipolar vague sets as follows;

\section{Definition 3.4: Let $\tilde{a}_{j}=$ $<\left[{T_{j}}^{-}, T_{j}^{+}\right]^{+},\left[I_{j}^{-}, I_{j}^{+}\right]^{+},\left[F_{j}^{-},{F_{j}}^{+}\right]^{+}$, \\ $\left[T_{j}^{-}, T_{j}^{+}\right]^{-},\left[I_{j}^{-}, I_{j}^{+}\right]^{-},\left[F_{j}^{-},{F_{j}}^{+}\right]^{-}>$ \\ $(j=1,2, \ldots, n)$ be a family of neutrosophic bipolar vague numbers. A mapping $A_{\omega}: \mathcal{Q}_{n} \rightarrow$ $\mathcal{Q}$ is called neutrosophic bipolar vague weighted average operator if it satisfies,}

$$
\begin{aligned}
& A_{\omega}\left(\tilde{a}_{1}, \tilde{a}_{2}, \tilde{a}_{3}, \ldots, \tilde{a}_{n}\right)=\sum_{j=1}^{n} \omega_{j} \tilde{a}_{j} \\
& =<1-\prod_{j=1}^{n}(1- \\
& \left.\left[{T_{j}}^{-}, T_{j}{ }^{+}\right]^{+}\right)^{\omega_{j}}, \prod_{j=1}^{n}\left(\left[I_{j}{ }^{-}, I_{j}^{+}\right]^{+}\right)^{\omega_{j}} \text {, } \\
& \prod_{j=1}^{n}\left(\left[F_{j}^{-}, F_{j}^{+}\right]^{+}\right)^{\omega_{j}} \text {, } \\
& -\prod_{j=1}^{n}\left(-\left[{T_{j}}^{-}, T_{j}{ }^{+}\right]^{-}\right)^{\omega_{j}} \text {, } \\
& -\left(1-\prod_{j=1}^{n}\left(1-\left(-\left[{I_{j}}^{-}, I_{j}{ }^{+}\right]^{-}\right)\right)^{\omega_{j}},\right. \\
& -\left(1-\prod_{j=1}^{n}\left(1-\left(-\left[{F_{j}}^{-},{F_{j}}^{+}\right]^{-}\right)\right)^{\omega_{j}}>\right.
\end{aligned}
$$

where $\omega_{j}$ is the weight of $\tilde{a}_{j}(\mathrm{j}=1,2 \ldots, \mathrm{n})$, $\omega_{j} \in[0,1]$ and $\sum_{j=1}^{n} \omega_{j}=1$.

Based on the study given in [ ] we give the theorem related to neutrosophic bipolar vague sets as follows;

Theorem

3.5:

$\tilde{a}_{j}=<\left[T_{j}{ }^{-},{T_{j}}^{+}\right]^{+},\left[I_{j}^{-}, I_{j}^{+}\right]^{+},\left[{F_{j}}^{-},{F_{j}}^{+}\right]^{+}$,
$\left[T_{j}^{-}, T_{j}^{+}\right]^{-},\left[I_{j}^{-}, I_{j}^{+}\right]^{-},\left[F_{j}^{-},{F_{j}}^{+}\right]^{-}>$

$(j=1,2 \ldots, n)$ be a family of neutrosophic bipolar vague numbers. Then,

i. If $\tilde{a}_{j}=\tilde{a}$ for all $\mathrm{j}=1,2, \ldots, \mathrm{n}$ then,

ii. $\quad \min _{j=1,2, \ldots, n}\left\{\tilde{a}_{j}\right\} \leq$ $A_{\omega}\left(\tilde{a}_{1}, \tilde{a}_{2}, \tilde{a}_{3}, \ldots, \tilde{a}_{n}\right)=\tilde{a}$.

$$
A_{\omega}\left(\tilde{a}_{1}, \tilde{a}_{2}, \tilde{a}_{3}, \ldots, \tilde{a}_{n}\right) \leq
$$$$
\max _{j=1,2, \ldots, n}\left\{\tilde{a}_{j}\right\}
$$

iii. If $\tilde{a}_{j} \leq \tilde{a}_{j}{ }^{*}$ for all $\mathrm{j}=1,2, \ldots, \mathrm{n}$ then,

$$
\begin{aligned}
& A_{\omega}\left(\tilde{a}_{1}, \tilde{a}_{2}, \tilde{a}_{3}, \ldots, \tilde{a}_{n}\right) \leq \\
& A_{\omega}\left(\tilde{a}_{1}{ }^{*}, \tilde{a}_{2}{ }^{*}, \tilde{a}_{3}{ }^{*}, \ldots, \tilde{a}_{n}{ }^{*}\right)
\end{aligned}
$$

Based on the study given in $[15,21]$ we define some weighted aggregation operators related to neutrosophic bipolar vague sets as follows;

Definition 3.6: Let $\tilde{a}_{j}=$ $<\left[{T_{j}}^{-}, T_{j}^{+}\right]^{+},\left[I_{j}^{-}, I_{j}^{+}\right]^{+},\left[{F_{j}}^{-},{F_{j}}^{+}\right]^{+}$,

$\left[T_{j}^{-}, T_{j}^{+}\right]^{-},\left[I_{j}^{-}, I_{j}^{+}\right]^{-},\left[F_{j}^{-},{F_{j}}^{+}\right]^{-}>$

$(j=1,2, \ldots, n)$ be a family of neutrosophic bipolar vague numbers. A mapping $G_{\omega}: \mathcal{Q}_{n} \rightarrow$ $\mathcal{Q}$ is called neutrosophic bipolar vague weighted geometric operator if it satisfies,

$$
\begin{aligned}
& G_{\omega}\left(\tilde{a}_{1}, \tilde{a}_{2}, \tilde{a}_{3}, \ldots, \tilde{a}_{n}\right)=\sum_{j=1}^{n} \omega_{j} \tilde{a}_{j} \\
& =<\prod_{j=1}^{n}\left(\left[T_{j}^{-}, T_{j}^{+}\right]^{+}\right)^{\omega_{j}}, \\
& 1-\prod_{j=1}^{n}\left(1-\left[I_{j}^{-}, I_{j}^{+}\right]^{+}\right)^{\omega_{j}}, 1- \\
& \prod_{j=1}^{n}\left(1-\left[F_{j}^{-}, F_{j}^{+}\right]^{+}\right)^{\omega_{j}},-(1- \\
& \prod_{j=1}^{n}\left(1-\left(-\left[T_{j}^{-}, T_{j}^{+}\right]^{-}\right)\right)^{\omega_{j}} \text {, } \\
& -\prod_{j=1}^{n}\left(-\left[I_{j}{ }^{-}, I_{j}{ }^{+}\right]^{-}\right)^{\omega_{j}},- \\
& \prod_{j=1}^{n}\left(-\left[F_{j}^{-},{F_{j}}^{+}\right]^{-}\right)^{\omega_{j}}>
\end{aligned}
$$

where $\omega_{j}$ is the weight of $\tilde{a}_{j}(\mathrm{j}=1,2 \ldots, \mathrm{n})$, $\omega_{j} \in[0,1]$ and $\sum_{j=1}^{n} \omega_{j}=1$.

Based on the study given in $[15,21]$ we give the theorem related to neutrosophic bipolar vague sets as follows;

Theorem

3.7:

Let

$\tilde{a}_{j}=<\left[T_{j}^{-}, T_{j}^{+}\right]^{+},\left[I_{j}^{-}, I_{j}^{+}\right]^{+},\left[F_{j}^{-}, F_{j}{ }^{+}\right]^{+}$,

\section{$\left[{T_{j}}^{-}, T_{j}^{+}\right]^{-},\left[I_{j}^{-}, I_{j}^{+}\right]^{-},\left[F_{j}^{-},{F_{j}}^{+}\right]^{-}>$}

$(j=1,2 \ldots, n)$ be a family of neutrosophic bipolar vague numbers. Then,

i. If $\tilde{a}_{j}=\tilde{a}$ for all $\mathrm{j}=1,2, \ldots, \mathrm{n}$ then,

ii. $\quad \min _{j=1,2, \ldots, n}\left\{\tilde{a}_{j}\right\} \leq$

$G_{\omega}\left(\tilde{a}_{1}, \tilde{a}_{2}, \tilde{a}_{3}, \ldots, \tilde{a}_{n}\right) \leq$ $\max _{j=1,2, \ldots, n}\left\{\tilde{a}_{j}\right\}$ 


\section{Available online at www.ijrat.org}

iii. If $\tilde{a}_{j} \leq \tilde{a}_{j}^{*}$ for all $\mathrm{j}=1,2, \ldots, \mathrm{n}$ then,

$$
\begin{aligned}
& G_{\omega}\left(\tilde{a}_{1}, \tilde{a}_{2}, \tilde{a}_{3}, \ldots, \tilde{a}_{n}\right) \leq \\
& G_{\omega}\left(\tilde{a}_{1}{ }^{*}, \tilde{a}_{2}{ }^{*}, \tilde{a}_{3}{ }^{*}, \ldots, \tilde{a}_{n}{ }^{*}\right) .
\end{aligned}
$$

Note that the aggregation results are still NBVNs.

\section{NBVN- DECISION MAKING METHOD}

In this section, we develop an approach based on the $A_{\omega}$ (or $G_{\omega}$ ) operator and the above ranking method to deal with multiple criteria decision making problems with neutrosophic bipolar vague information.

Suppose that $A=\left\{A_{1}, A_{2}, \ldots, A_{m}\right\}$ and $C=\left\{C_{1}, C_{2}, \ldots, C_{n}\right\}$ is the set of alternatives and criterions or attributes, respectively. Let $\omega=\left(\omega_{1}, \omega_{2}, \ldots, \omega_{n}\right)^{T}$ be the weight vector of attributes, such that $\sum_{j=1}^{n} \omega_{j}=1, \omega_{j} \geq 0$ $(j=1,2, \ldots, n)$ and $\omega_{j}$ refers to the weight of attribute $C_{j}$. An alternative on criterions is evaluated by the decision maker, and the evaluation values are represented by the form of neutrosophic bipolar vague numbers. Assume that,

$\left(\widetilde{a_{l \jmath}}\right)_{m \times n}=$

$\left(<\left[T_{i j}{ }^{-}, T_{i j}{ }^{+}\right]^{+},\left[I_{i j}{ }^{-}, I_{i j}{ }^{+}\right]^{+},\left[F_{i j}{ }^{-}, F_{i j}{ }^{+}\right]^{+}\right.$,

$\left.\left[T_{i j}{ }^{-}, T_{i j}{ }^{+}\right]^{-},\left[I_{i j}{ }^{-}, I_{i j}{ }^{+}\right]^{-},\left[F_{i j}{ }^{-},{F_{i j}}^{+}\right]^{-}>\right)_{m \times n}$ is the decision matrix provided by the decision maker; $\widetilde{a_{\imath \jmath}}$ is a neutrosophic bipolar vague number for alternative $A_{i}$ associated with the criterions $C_{j}$. We have the conditions $\left[T_{i j}{ }^{-}, T_{i j}{ }^{+}\right]^{+},\left[I_{i j}{ }^{-}, I_{i j}{ }^{+}\right]^{+},\left[F_{i j}{ }^{-},{F_{i j}}^{+}\right]^{+}$,

$\left[T_{i j}{ }^{-}, T_{i j}{ }^{+}\right]^{-},\left[I_{i j}{ }^{-}, I_{i j}{ }^{+}\right]^{-},\left[F_{i j}{ }^{-}, F_{i j}{ }^{+}\right]^{-} \in[0,1]$ such that

$$
\begin{aligned}
& 0 \leq\left[T_{i j}{ }^{-}, T_{i j}{ }^{+}\right]^{+}+\left[I_{i j}{ }^{-}, I_{i j}{ }^{+}\right]^{+}+ \\
& {\left[F_{i j}{ }^{-},{F_{i j}}^{+}\right]^{+}-\left[T_{i j}{ }^{-},{T_{i j}}^{+}\right]^{-}-\left[I_{i j}{ }^{-}, I_{i j}{ }^{+}\right]^{-}-} \\
& {\left[F_{i j}{ }^{-}, F_{i j}{ }^{+}\right]^{-} \leq 6 \text { for } \mathrm{i}=1,2, \ldots, \mathrm{m} \text { and }} \\
& \mathrm{j}=1,2, \ldots, \mathrm{n} .
\end{aligned}
$$

Now, we develop an algorithm as follows:

\section{Algorithm}

Step 1: Construct the decision matrix provided by the decision maker as,

$$
\begin{aligned}
& \left.\left(\widetilde{a_{\imath j}}\right)_{m \times n}-T_{i j}{ }^{+}\right]^{+},\left[I_{i j}{ }^{-}, I_{i j}{ }^{+}\right]^{+},\left[F_{i j}{ }^{-}, F_{i j}{ }^{+}\right]^{+}, \\
& =\begin{array}{l}
\left(<\left[T_{i j},{ }^{-}\right.\right. \\
\left.\left[T_{i j}{ }^{-}, T_{i j}{ }^{+}\right]^{-},\left[I_{i j}{ }^{-}, I_{i j}{ }^{+}\right]^{-},\left[F_{i j}{ }^{-}, F_{i j}{ }^{+}\right]^{-}>\right)_{m \times n}
\end{array}
\end{aligned}
$$

Step 2: $\quad$ Compute $\quad \tilde{a}_{i}=$ $A_{\omega}\left(\tilde{a}_{i 1}, \tilde{a}_{i 2}, \tilde{a}_{i 3}, \ldots, \tilde{a}_{i n}\right)\left(\right.$ or $\left.G_{\omega}\left(\tilde{a}_{i 1}, \tilde{a}_{i 2}, \tilde{a}_{i 3}, \ldots, \tilde{a}_{i n}\right)\right)$ for each $i=1,2 \ldots, m$.

Step 3: Calculate the score values of $\tilde{s}\left(\widetilde{a_{l}}\right)$ $(\mathrm{i}=1,2, \ldots \mathrm{m})$ for the collective overall neutrosophic bipolar vague

number of $\tilde{a}_{i}(\mathrm{i}=1,2, \ldots, \mathrm{m})$

Step 4: Rank all the software systems of $\tilde{a}_{i}(\mathrm{i}=1,2, \ldots, \mathrm{m})$ according to the score values.

Now, we give a numerical example as follows:

Example 4.1: Let us consider the decision making problem adapted from $\mathrm{Xu}$ and $\mathrm{Cia}[21]$. A customer who intends to buy a car. Four types of cars (alternatives) $A_{i}(\mathrm{i}=1,2,3,4)$ are available. The customer takes into ccount four attributes to evaluate the alternatives; $C_{1}=$ Fuel Economy; $C_{2}=$ Aerod; $C_{3}=$ Comfort; $C_{4}=$ Safety and the use of neutrosophic bipolar vague values to evaluate the four possible alternatives $A_{i}(\mathrm{i}=1,2,3,4)$ under the above four attributes. Also, the weight vector of the attributes $C_{j}(\mathrm{j}=1,2,3,4)$ is $\omega=\left(\frac{1}{2}, \frac{1}{4}, \frac{1}{8}, \frac{1}{8}\right)^{T}$. Then,

\section{Algorithm}

Step 1: Construct the decision matrix provided by the customer as;

\begin{tabular}{|c|c|c|c|c|}
\hline & $C_{1}$ & $C_{2}$ & $C_{3}$ & $C_{4}$ \\
\hline \multirow{2}{*}{$A_{1}$} & {$[0.4,0.5][0.5,0.5]$} & {$[0.4,0.6][0.2,0.2]$} & {$[0.2,0.5][0.5,0.5]$} & {$[0.4,0.5][0.5,0.5]$} \\
& {$[0.5,0.6][-0.6,-0.4]$} & {$[0.4,0.6][-0.5,-0.3]$} & {$[0.5,0.8][-0.4,-0.1]$} & {$[0.5,0.6][-0.3,-0.2]$} \\
& {$[-0.3,-0.3][1.4,1.6]$} & {$[-0.6,-0.6][1.3,1.5]$} & {$[-0.5,-0.6][1.1,1.4]$} & {$[-0.2,-0.2][1.2,1.3]$} \\
\hline \multirow{2}{*}{$A_{2}$} & {$[0.7,0.9][0.7,0.7]$} & {$[0.4,0.7][0.8,0.4]$} & {$[0.6,0.7][0.5,0.8$} & {$[0.2,0.5][0.2,0.2]$} \\
& {$[0.1,0.3][-0.3,-0.2]$} & {$[0.3,0.6][-0.4,-0.3]$} & ]$[0.3,0.4][-0.1,-0.1]$ & {$[0.5,0.8][-0.5,-0.4]$} \\
& {$[-0.7,-0.7][1.2,1.3]$} & {$[-0.4,-0.4][1.3,1.4]$} & {$[-0.6,-0.6][1.1,1.1]$} & {$[-0.2,-0.1][1.4,1.5]$} \\
\hline \multirow{2}{*}{$A_{3}$} & {$[0.5,0.7][0.5,0.5]$} & {$[0.3,0.6][0.4,0.4]$} & {$[0.7,0.8][0.6,0.3]$} & {$[0.6,0.7][0.5,0.5]$} \\
& {$[0.3,0.5][-0.4,-0.1]$} & {$[0.4,0.7][-0.2,-0.2]$} & {$[0.2,0.3][-0.5,-0.3]$} & {$[0.3,0.4][-0.1,-0.2]$} \\
& {$[-0.5,-0.6][1.1,1.4]$} & {$[-0.8,-0.6][1.2,1.2]$} & {$[-0.7,-0.7][1.3,1.5]$} & {$[-0.6,-0.6][1.2,1.1]$} \\
\hline \multirow{2}{*}{$A_{4}$} & {$[0.5,0.9][0.3,0.3]$} & {$[0.4,0.6][0.2,0.2]$} & {$[0.3,0.5][0.7,0.8]$} & {$[0.4,0.7][0.6,0.8]$} \\
& {$[0.1,0.5][-0.4,-0.3]$} & {$[0.4,0.6][-0.5,-0.3]$} & {$[0.5,0.7][-0.5,-0.1]$} & {$[0.3,0.6][-0.4,-0.2]$} \\
& {$[-0.4,-0.4][1.3,1.4]$} & {$[-0.5,-0.5][1.3,1.5]$} & {$[-0.3,-0.6][1.1,1.5]$} & {$[-0.3,-0.5][1.2,1.4]$} \\
\hline
\end{tabular}




\section{Available online at www.ijrat.org}

Step 2: Compute $\tilde{a}_{i}=A_{\omega}\left(\tilde{a}_{i 1}, \tilde{a}_{i 2}, \tilde{a}_{i 3}, \tilde{a}_{i 4}\right)$ for each $\mathrm{i}=1,2,3,4$ as;

$\tilde{a}_{1}=[0.3782,0.5271],[0.3866,0.3866]$,

[0.4728,0.6217], [-0.4748,-0.3018],

[-0.4067,-0.4231], [1.3223,1.4968]

$\tilde{a}_{2}=[0.5820,0.8145],[0.6290,0.4988]$,

[0.1845,0.4179], [-0.2990,-0.2212],

[-0.5758,-0.5820], [1.2353,1.3218]

$\tilde{a}_{3}=[0.5039,0.6936],[0.4826,0.4435]$,

[0.3063,0.4960], [-0.2907,-0.1487],

[-0.5687,-0.6756], [1.1609,1.6024]

$\tilde{a}_{4}=[0.4417,0.8016],[0.3285,0.3463]$,

[0.1983,0.5582], [-0.3260,-0.3314],

[-0.4044,-0.4675], [1.3359,1.3581]

Step 3: Calculate the score values $\tilde{s}\left(\widetilde{a_{l}}\right)(\mathrm{i}=1,2,3,4)$ for the collective overall neutrosophic bipolar vague number of $\left.\widetilde{a_{l}}\right)(\mathrm{i}=1,2,3,4)$ as;

$\tilde{s}\left(\widetilde{a_{1}}\right)=0.1895$

$\tilde{s}\left(\widetilde{a_{2}}\right)=0.3123$

$$
\begin{aligned}
& \tilde{s}\left(\widetilde{a_{3}}\right)=0.2925 \\
& \tilde{s}\left(\widetilde{a_{4}}\right)=0.2777
\end{aligned}
$$

Step 4: Rank all the software systems of $A_{i}$ $(i=1,2,3,4)$ according to the score values as;

$A_{2}>A_{3}>A_{4}>A_{1}$

And thus $A_{2}$ is the most desirable alternative.

Let us discuss another numerical example for easy understanding:

\section{Example 4.2:}

People who are affected by cancer, have a combination of treatments, such as surgery with chemotherapy and/or radiation therapy, hormone therapy, and immunotherapy. Our main objective is to find the best treatment from the above mentioned therapies. However, all the treatments can cause side effects. Our goal is to find the best treatment which cause the least side effects, extend the patient's life, cure the cancer and control its growth using an Neutrosophic Bipolar Vague set. Also the weight vector of the attributes $C_{j}(\mathrm{j}=1,2,3,4$, is $\omega=(0.08,0.62,0.05,0.25)^{T}$.

\section{Algorithm}

\begin{tabular}{|c|c|c|c|c|}
\hline & $\mathrm{C}_{1}$ & $\mathrm{C}_{2}$ & $\mathrm{C}_{3}$ & $\mathrm{C}_{4}$ \\
\hline \multirow[t]{3}{*}{$\mathrm{A}_{1}$} & {$[0.2,0.8][0.1,0.1]$} & {$[0.4,0.6][0.3,0.3]$} & {$[0.4,0.4][0.1,0.2]$} & {$[0.4,0.5][0.3,0.7]$} \\
\hline & {$[0.2,0.8][-0.6,-0.4]$} & {$[0.4,0.6][-0.3,-0.2]$} & {$[0.6,0.6][-0.7,-0.3]$} & {$[0.5,0.6][-0.7,-0.4]$} \\
\hline & {$[-0.2,-0.2][1.4,1.6]$} & {$[-0.3,-0.3][1.2,1.3]$} & {$[-0.6,-0.2][1.3,1.7]$} & {$[-0.5,-0.1][1.4,1.7]$} \\
\hline \multirow[t]{3}{*}{$\mathrm{A}_{2}$} & {$[0.1,0.2][0.4,0.4]$} & {$[0.3,0.4][0.6,0.6]$} & {$[0.5,0.7][0.2,0.3]$} & {$[0.3,0.6][0.4,0.5]$} \\
\hline & {$[0.8,0.9][-0.4,-0.3]$} & {$[0.6,0.7][-0.5,-0.4]$} & {$[0.3,0.5][-0.5,-0.3]$} & {$[0.4,0.7][-0.4,-0.2]$} \\
\hline & {$[-0.7,-0.7][1.3,1.4]$} & {$[-0.1,-0.1][1.4,1.5]$} & {$[-0.3,-0.2][1.3,1.5]$} & {$[-0.6,-0.3][1.2,1.4]$} \\
\hline \multirow[t]{3}{*}{$\mathrm{A}_{3}$} & {$[0.4,0.5][0.5,0.5]$} & {$[0.5,0.6][0.7,0.7]$} & {$[0.2,0.7][0.1,0.4]$} & {$[0.3,0.7][0.2,0.6]$} \\
\hline & {$[0.5,0.6][-0.4,-0.3]$} & {$[0.4,0.5][-0.6,-0.3]$} & {$[0.3,0.8][-0.6,-0.4]$} & {$[0.3,0.7][-0.5,-0.5]$} \\
\hline & {$[-0.6,-0.6][1.3,1.4]$} & {$[-0.4,-0.3][1.3,1.6]$} & {$[-0.5,-0.2][1.4,1.6]$} & {$[-0.6,-0.4][1.5,1.5]$} \\
\hline \multirow[t]{3}{*}{$\mathrm{A}_{4}$} & {$[0.2,0.3][0.2,0.2]$} & {$[0.2,0.4][0.3,0.4]$} & {$[0.6,0.8][0.3,0.4]$} & {$[0.3,0.6][0.5,0.6]$} \\
\hline & {$[0.7,0.8][-0.3,-0.2]$} & {$[0.6,0.8][-0.5,-0.4]$} & {$[0.2,0.4][-0.4,-0.1]$} & {$[0.4,0.7][-0.5,-0.4]$} \\
\hline & {$[-0.4,-0.4][1.2,1.3]$} & $[-0.1,-0.2]][1.4,1.5]$ & {$[-0.5,-0.3][1.1,1.4]$} & {$[-0.3,-0.1][1.4,1.5]$} \\
\hline
\end{tabular}

Step 1: Construct the decision matrix provided by the customer as;

Step 2: Compute $\tilde{a}_{i}=A_{\omega}\left(\tilde{a}_{i 1}, \tilde{a}_{i 2}, \tilde{a}_{i 3}, \tilde{a}_{i 4}\right)$ for

$[0.5357,0.7021][-0.4643,-0.3216]$

each $\mathrm{i}=1,2,3,4$ as;

$\tilde{a}_{1}=[0.3861, .05917][0.2599,0.3326]$

[0.4082,0.6138][-0.4087,-0.2564]

[-0.3676,-0.2415][1.2688.1.4367]

$\tilde{a}_{2}=[0.2978,0.4642][0.4966,0.5359]$

$[-0.3355,0.2306][1.3351,1.4661]$

$\tilde{a}_{3}=[0.4351,0.6265][0.4519,0.6374]$

[0.3734.0.5648][-0.5548,-0.3456]

$[-0.4800,-0.3516][1.3531,1.5578]$ 
$\tilde{a}_{4}=[0.2527,0.4806][0.3298,0.4187]$

$[0.5193,0.7472][-0.4744,-0.3529]$

\section{$[-0.2056,-0.2003][1.3671,1.4781]$}

Step 3: Calculate the score values $\tilde{s}\left(\widetilde{a_{l}}\right)(\mathrm{i}=1,2,3,4)$ for the collective overall neutrosophic bipolar vague number of $\left(\widetilde{a_{l}}\right)(\mathrm{i}=1,2,3,4)$ as;

$\tilde{s}\left(\widetilde{a_{1}}\right)=0.2168$

$\tilde{s}\left(\widetilde{a_{2}}\right)=0.1225$

$\tilde{s}\left(\widetilde{a_{3}}\right)=0.1712$

$\tilde{s}\left(\widetilde{a_{4}}\right)=0.1209$

Step 4: Rank all the software systems of $A_{i}$ $(i=1,2,3,4)$ according to the score values as;

$A_{1}>A_{3}>A_{2}>A_{4}$

Thus $A_{1}$ is the most desirable alternative. (i.e.,) the best treatment for cancer patients is chemotherapy or radiation therapy.

\section{CONCLUSIONS}

This paper presented a neutrosophic bipolar vague set and its score, certainty and accuracy functions. Then, the $A_{\omega}$ and $G_{\omega}$ operators were proposed to aggregate the neutrosophic bipolar vague information. Furthermore, based on the $A_{\omega}$ and $G_{\omega}$ operators and the score, certainty and accuracy functions, we have developed a neutrosophic bipolar vague multiple criteria decision-making approach, in which the evaluation values of alternatives on the attributes take the form of neutrosophic bipolar vague numbers. The $A_{\omega}$ and $G_{\omega}$ operators are utilized to aggregate the neutrosophic bipolar vague information corresponding to each alternative to obtain the collective overall values of the alternatives, and then the alternatives are ranked according to the values of the score, certainty and accuracy functions to select the most desirable one(s). Finally, a numerical example of the method was given to demonstrate the application and effectiveness of the developed method.

\section{REFERENCES}

[1] K. Atanassov, Intuitionistic fuzzy sets. Fuzzy Sets and Systems, 20 (1986) 87-96.

[2] P. Bosc, O. Pivert, On a fuzzy bipolar relational algebra, Information Sciences, 219 (2013) 1- 16.

[3] H. D. Cheng and Y. Guo, A new neutrosophic approach to image thresholding, New Mathematics and Natural Computation, 4(3) (2008) 291-308.
[4]Gau. W. L, Buehrer. D.J. 1993. Vague sets, IEEE Transcations on Systems, Man and Cybernatics, 23(2), 610-614.

[5] Y. Guo and H. D. Cheng, New Neutrosophic Approach to Image Segmentation, Pattern Recognition, 42, (2009), 587-595.

[6] Irfan Deli, Mumtaz Ali and Florentin Smarandache, Bipolar Neutrosophic Sets And Their Application Based on Multi-Criteria Decision Making Problems. In Proceedings of the 2015 International Conference on Advanced Mechatronic Systems, Beiging, China, 20-24 August 2015; pp. 249-254.

[7] A. Kharal, A neutrosophic multicriteria decision making method, New Mathematics \& Natural Computation, 2013.

[8] M. K. Kang and J. G. Kang, Bipolar fuzzy set theory applied to sub-semigroups with operators in semigroups. J. Korean Soc. Math. Educ. Ser. B Pure Appl. Math., 19/1 (2012) 2335.

[9] K. M. Lee, Bipolar-valued fuzzy sets and their operations. Proc. Int. Conf. on Intelligent Technologies, Bangkok, Thailand (2000) 307312.

[10] K. J. Lee, Bipolar fuzzy subalgebras and bipolar fuzzy ideals of BCK/BCI-algebras, Bull. Malays. Math. Sci. Soc., 32/3 (2009) 361-373.

[11] P. Liu and Y. Wang, Multiple attribute decision-making method based on singlevalued neutrosophic normalized weighted Bonferroni mean, Neural Computing and Applications, 25 7/8 (2014) 2001-2010.

[12] P. Liu L. Shi, The generalized hybrid weighted average operator based on interval neutrosophic hesitant set and its application to multiple attribute decision making, Neural Computing and Applications, 26 /2 (2015) 457-471.

[13] P. Majumdar and S.K. Samanta, On similarity and entropy of neutrosophic sets, J. Intell. Fuzzy Syst. 26/3 (2014) 1245-1252.

[14]Mohana K, Princy R, Florentin Smarandache, "An Introduction to neutrosophic bipolar vague topological spaces". [communicated].

[15]J.J. Peng, J.Q. Wang, J. Wang, H.Y. Zhang and X.H. Chen, Simplified neutrosophic sets and their applications in multi-criteria group decision-making problems, Int. J. Syst. Sci.

(2015) DOI:10.1080/00207721.2014.994050.

[16] R. Sahin and A. Kucuk, Subsethood measure for single valued neutrosophic sets, Journal of Intelligent and Fuzzy Systems, (2014) DOI: 10.3233/IFS-141304.

[17]Shawkat Alkhazaleh, Neutrosophic vague set theory, Critical Review, Vol X, 2015 
[18] F. Smarandache, A Unifying Field in Logics. Neutrosophy : Neutrosophic Probability, Set and Logic, Rehoboth: American Research Press, 1999.

[19] H. Wang, F. Smarandache, Y.Q. Zhang and R. Sunderraman Single valued neutrosophic sets, Multispace and Multistructure, 4 (2010) $10-413$

[20] H. Wang, F. Smarandache, Y.Q. Zhang and R. Sunderraman, Interval neutrosophic sets and logic: theory and applications in computing, (2005) Hexis, Arizona.

[21] Z. Xu, X. Cai, Intuitionistic Fuzzy Information Aggregation Theory and Applications, Springer, Science Press, Heidelberg New York Dordrecht London, 2011.

[22] J. Ye Multicriteria decision-making method using the correlation coefficient under single valued neutrosophic environment, International Journal of General Systems 42/4 (2013) 386394.

[23] J. Ye, Similarity measures between interval neutrosophic sets and their applications in Multi-criteria decision-making. Journal of Intelligent and Fuzzy Systems, 26 (2014) 165 172.

[24] J. Ye, Single valued neutrosophic crossentropy for multi-criteria decision making problems, Appl. Math.Model. 38 /3 (2014) 1170-1175.

[25] J. Ye, Trapezoidal neutrosophic set and its application to multiple attribute decision making, Neural Computing and Applications, (2014) DOI: 10.1007/s00521-014-1787-6

[26] J. Ye, Some aggregation operators of interval neutrosophic linguistic numbers for multiple attribute decision making, Journal of Intelligent \& Fuzzy Systems 27 (2014) 22312241

[27] L.A. Zadeh, Fuzzy sets, Inf. Control, 8 (1965) 338-353.

[28] H.Y. Zhang, J.Q. Wang, X.H. Chen, Interval neutrosophic sets and their application in multicriteria decision making problems. The Scientific World Journal. (2014) DOI: $10.1155 / 2014 / 645953$.

[29] M. Zhang, L. Zhang, and H.D. Cheng. A Neutrosophic Approach to Image Segmentation based on Watershed Method, Signal Processing 5/ 90 (2010) 1510-1517. 